\title{
Peritumoral edema on magnetic resonance imaging predicts a poor clinical outcome in malignant glioma
}

\author{
CHEN-XING WU ${ }^{1 *}$, GUO-SHI LIN ${ }^{2 *}$, ZHI-XIONG LIN ${ }^{1,3}$, JIAN-DONG ZHANG $^{3}$, LONG CHEN $^{3}$, \\ SHUI-YUAN LIU ${ }^{3}$, WEN-LONG TANG ${ }^{3}$, XIAN-XIN QIU ${ }^{3}$ and CHANG-FU ZHOU ${ }^{2}$ \\ ${ }^{1}$ Department of Neurosurgery, Beijing Sanbo Brain Hospital, Capital Medical University, Beijing 100093; \\ ${ }^{2}$ Department of Neurosurgery, Zhangzhou Affiliated Hospital of Fujian Medical University, Zhangzhou, Fujian 363000; \\ ${ }^{3}$ Department of Neurosurgery, The First Affiliated Hospital of Fujian Medical University, Fuzhou, Fujian 350005, P.R. China
}

Received October 12, 2014; Accepted August 5, 2015

DOI: $10.3892 / \mathrm{ol} .2015 .3639$

\begin{abstract}
Peritumoral edema (PTE), one of the main characteristics of malignant glioma, is a significant contributor to the morbidity and mortality from glioma, however, a recent systematic review suggested that controversy remains with regard to its prognostic value. To further determine whether PTE was a potential prognostic factor on routine pre-operative magnetic resonance imaging (MRI) for malignant glioma, the association between survival and PTE was investigated in the present retrospective review of 109 patients with newly diagnosed supratentorial malignant glioma using MRI data from these routine scans. The Kaplan-Meier method was used to calculate overall survival (OS) in univariate analysis, and COX proportional hazards model was applied to evaluate the effect of pre-operative MRI features on OS in multivariate analysis. The PTE extent, edema shape, degree of necrosis, enhancement extent, pathological grade, patient age, Karnofsky performance status (KPS) and post-operative chemoradiotherapy were associated with OS in the patients with malignant glioma on univariate analysis. Multivariate analysis indicated that the extent of PTE and degree of necrosis shown by pre-operative MRI were independent predictors of OS, in addition to pathological grade, patient age, KPS and post-operative chemoradiotherapy. Moreover, patients with two unfavorable factors (major edema and severe necrosis) exhibited a poorer OS compared with the remainder. In summary, PTE and degree of necrosis, which are easily determined from routine MRI, can be useful for predicting a poor clinical outcome in patients with newly diagnosed malignant glioma.
\end{abstract}

Correspondence to: Dr Zhi-Xiong Lin, Department of Neurosurgery, The First Affiliated Hospital of Fujian Medical University, 20 Cazhong Road, Fuzhou, Fujian 350005, P.R. China

E-mail: 1zx1967@sina.com

*Contributed equally

Key words: malignant glioma, glioblastoma, survival, prognosis, peritumoral edema, magnetic resonance imaging

\section{Introduction}

Malignant glioma, which consists mainly of anaplastic glioma and glioblastoma (GBM), represents up to $50 \%$ of all primary brain gliomas (1) and is the most common primary brain tumor in adults. The clinical outcome in patients with malignant glioma is poor despite the improvements in survival rate since the use of adjuvant chemoradiotherapy post-operatively (2).

It has been reported that the prognosis of malignant glioma may be affected by several factors, including not only patient age, pre-operative Karnofsky performance status (KPS), histological grade, pathological molecular markers (such as MGMT, IDH1, 1p19q and PDGF- $\alpha$ ) and temozolomide chemotherapy (3-7), but also certain pre-operative magnetic resonance imaging (MRI) features on routine scans, such as peritumoral edema (PTE) extent, degree of necrosis, enhancement extent and the size of the cyst, among others (5,8-11). However, several studies have also shown that certain features of pre-operative MRI, such as PTE, cysts and tumor size, are not independent predictors of survival in patients with glioma (12-14).

PTE is a significant contributor to morbidity and mortality from glioma (15), but a recent systematic review suggested that controversy remains with regard to its prognostic value (14). A requirement therefore exists to further evaluate the association between PTE on MRI and patient survival, as such data from routine imaging are irreplaceable during the formation of a pre-operative diagnosis and are now the central basis of treatment decisions in patients with glioma. Determination of clear associations between these factors has a certain instructive significance for clinical practice. In the present study, the prognostic value of MRI features from pre-operative routine scans was assessed in patients with malignant glioma in order to confirm which would be the most valuable prognostic markers, as this data may provide aid in everyday clinical activities.

\section{Patients and methods}

Patients and clinical data. The clinical and pre-operative MRI data of 109 patients treated by resection of a newly diagnosed supratentorial malignant glioma at the First Affiliated Hospital of Fujian Medical University (Fuzhou, China) between March 2006 and September 2012 were 

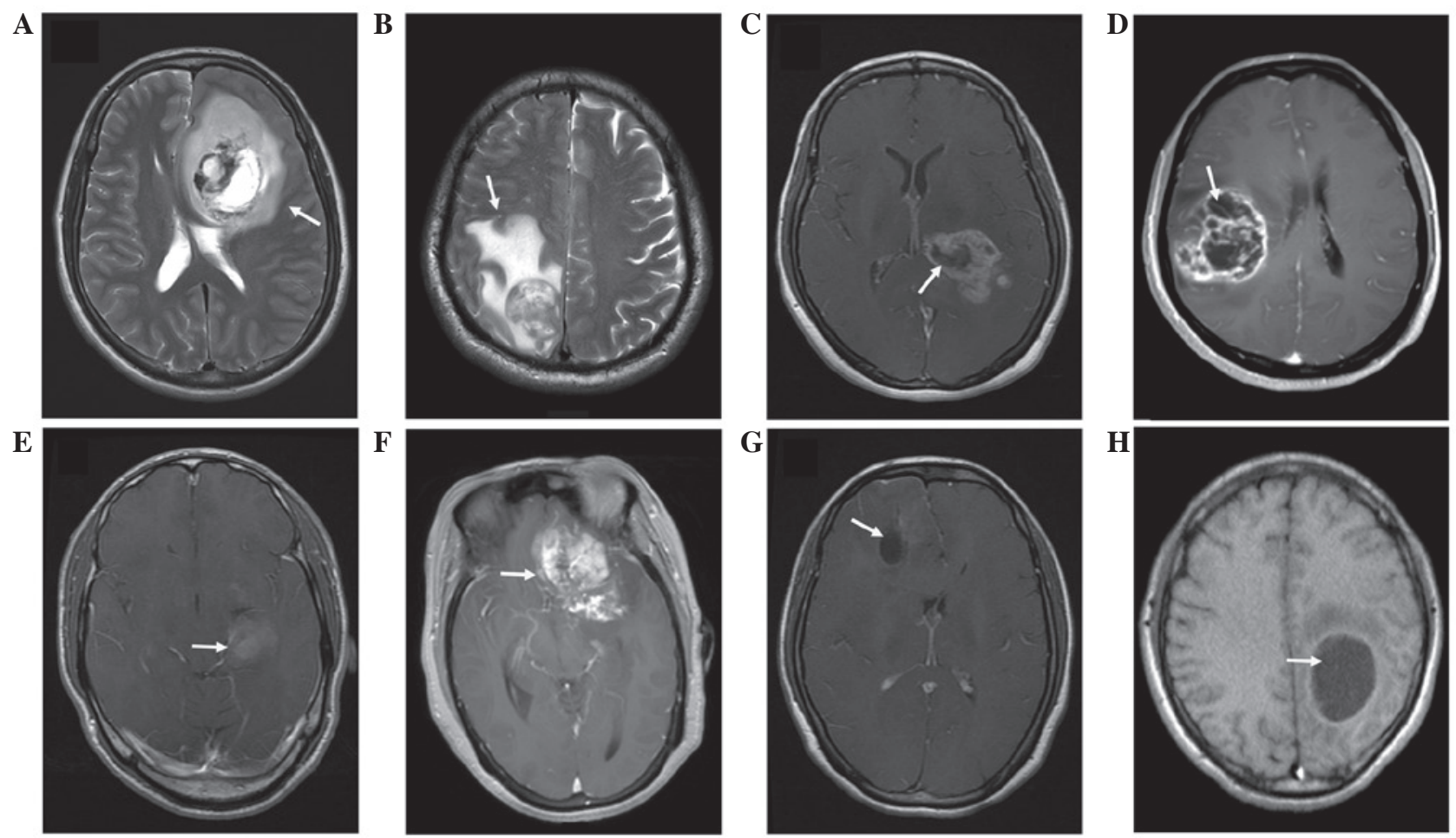

Figure 1. Example of classification of imaging features (arrows). (A) Minor edema $(<1 \mathrm{~cm})$ and the rounded edema shape shown by T2-W MRI. (B) Major edema $(\geq 1 \mathrm{~cm})$ and the irregular shape of edema shown by T2-W MRI. (C-H) Contrast-enhanced T1-W images showing (C) mild necrosis $(\leq 50 \%),(\mathrm{D})$ severe necrosis $(>50 \%)$, (E) no marked enhancement, (F) marked enhancement, $(\mathrm{G})$ a small cyst and $(\mathrm{H})$ a large cyst. T2-W MRI, T2-weighted magnetic resonance imaging.

included in this retrospective study. Patients who succumbed to non-glioma-based causes or those who received a biopsy were excluded from the study. No patients received corticosteroids at the time of the pre-operative MRI scan. For all patients, the tumor was confirmed to be totally resected using post-operative enhanced MRI within 3 days. According to the principles of the World Health Organization classification (16), the histological diagnosis and grading of each patient were reaffirmed. In total, 65 cases were classified as GBM (grade 4) and 44 as anaplastic glioma (grade 3), consisting of anaplastic astrocytoma, anaplastic oligodendroglioma and anaplastic oligoastrocytoma. In the cohort, 65 patients were male and 44 were female. The median age at diagnosis was 49 years (range, 18-78 years). The median pre-operative KPS of the patients was 80 (range, 70-100). Post-operatively, 53 patients were treated with standard chemoradiotherapy (radiotherapy plus chemotherapy) and 56 were treated with non-standard chemoradiotherapy (17) (radiotherapy alone, chemotherapy alone, and no radiotherapy or chemotherapy). Oral temozolomide chemotherapy $\left(150-200 \mathrm{mg} / \mathrm{m}^{2} /\right.$ day) was administered for 4-6 cycles unless mortality or irreversible hematological toxicity occurred. Radiotherapy was administered to the contrast-enhanced lesion plus the area of the PTE and a 2-cm margin (60 Gy in 2-Gy fractions). All patients were followed up via telephone conversations or outpatient visits. Overall survival (OS) was defined as the time (months) between the primary surgical resection and mortality or the latest follow-up. This study was approved by Ethics Committee of Fujian Medical University and conformed to the principles outlined in the Declaration of Helsinki. Written informed consent was provided by all patients.
Classification of MRI features. For all patients, pre-operative MRI data from routine scans, including T1-weighted (W), T2-W and contrast-enhanced T1-W sequences were available. The unidimensional maximum diameter $(\mathrm{cm})$ was used for measuring the tumor size on the T1-W images, and the median tumor size was recorded as $5.0 \mathrm{~cm}$ (range, 2.3-9.0 cm). The bright T2-W signal region surrounding the tumor was defined as PTE, which was estimated based on the maximum distance from the tumor margin to the outer edge of the edema, and was graded into minor (Fig. 1A) and major (Fig. 1B) types (5). According to the method reported by Hartmann et al (18), the morphological classification of PTE was performed on the basis of the T2-W images (Fig. 1A and B). Necrosis, which was estimated on the axial contrast-enhanced T1-W images (19), was demonstrated when a region had a high signal intensity on T2-W images, but a low signal intensity on T1-W images, and an irregular enhancing border on contrast-enhanced images (Fig. 1C and D). Enhancement was defined as not marked or marked when the enhancement signal was less than (Fig. 1E) or similar to (Fig. 1F) the signal intensity of fat, respectively. Cysts (Fig. 1G and $\mathrm{H}$ ) were defined as rounded regions that exhibited low intensity T1-W signals and extremely high intensity T2-W signals matching the cerebrospinal fluid signal. Additionally, the regions presented with a thin, smooth, regular, slightly enhancing or non-enhancing wall (9). The specific classification of the imaging features is listed in Table I and an example of this classification is presented in Fig. 2. According to these aforementioned classification methods, the imaging data of all the patients were analyzed independently by two experienced radiologists who were blinded to the patient's clinical information. 
Table I. Specific classification of imaging features.

$\begin{array}{ll}\text { Imaging features } & \text { Classification criteria }\end{array}$

PTE
Minor
Major
Edema shape
Rounded
Irregular
Necrosis
No
Mild
Severe
Cyst
No
Small
Large
Enhancement
No marked
Marked

Tumor crosses midline

No

Yes

Edema crosses midline

No

Yes

Size, $\mathrm{cm}$

$<5$

$\geq 5$
PTE extending $<1 \mathrm{~cm}$ from tumor margin

PTE extending $\geq 1 \mathrm{~cm}$ from tumor margin

The edema shape is rounded and is not radial

The edema shape tends to be irregular, e.g., finger-like or radial shapes

No necrosis within the tumor

Necrosis affecting $\leq 50 \%$ of the tumor

Necrosis affecting $>50 \%$ of the tumor

No cyst in the tumor

Cyst cavity in $\leq 50 \%$ of the total tumor

Cyst cavity in $>50 \%$ of the total tumor

Enhancement signal is less than the signal intensity of fat

Enhancement signal is similar to the signal intensity of fat

Tumor is limited to the unilateral cerebral hemisphere

Tumor crosses the brain midline and extends into the other side of the cerebral hemisphere

PTE extent is limited to the unilateral cerebral hemisphere

PTE extent crosses the brain midline and is not confined to the unilateral cerebral hemisphere

The maximum diameter of the tumor is $<5 \mathrm{~cm}$

The maximum diameter of the tumor is $\geq 5 \mathrm{~cm}$
Statistical analysis. SPSS 19.0 (IBM SPSS, Armonk, NY, USA) was applied for the statistical analysis. For the univariate analysis, the Kaplan-Meier method was used to calculate survival rates, which were compared by the log-rank test. Factors that were statistically significant on the univariate analysis were analyzed on multivariate analysis. COX proportional hazards model and stepwise regression analysis were applied to estimate the effect of pre-operative MRI features on survival in the multivariate analysis. $\mathrm{P} \leq 0.05$ (two-sided) was considered to indicate a statistically significant difference.

\section{Results}

PTE. Univariate analysis (Table II) showed that patients with major PTE survived a significantly shorter amount of time than those with minor PTE $(\mathrm{P}<0.0001$; Fig. $2 \mathrm{~A})$; the median OS time was 14.0 and 44.0 months, respectively. Multivariate analysis (Table III) indicated that the PTE extent shown by pre-operative MRI was an independent prognostic factor in patients with malignant glioma $[\mathrm{P}=0.029$; hazard ratio (HR), 2.337].
Necrosis. The degree of necrosis on pre-operative MRI was also an independent prognostic factor in malignant glioma $(\mathrm{P}<0.0001 ; \mathrm{HR}, 2.218)$, with a median OS time of 42.0 , 14.0 and 10.0 months for patients with no, mild and severe necrosis, respectively ( $\mathrm{P}<0.0001$; Fig. $2 \mathrm{~B})$.

Additionally, the prognosis in the patients with two unfavorable factors (major edema and severe necrosis) was markedly poor compared with those with only one unfavorable factor and those without unfavorable factors ( $\mathrm{P}<0.0001$; Fig $2 \mathrm{E})$, with median OS times of 10.0, 15.0 and 44.0 months, respectively.

Other imaging features. On univariate analysis, a significant difference $(\mathrm{P}<0.0001$; Fig. $2 \mathrm{C})$ was indicated between the patients with no marked enhancement and those with marked enhancement, with median OS times of 29.0 and 13.0 months, respectively. However, multivariate analysis showed that the extent of enhancement was not an independent prognostic factor. Similarly, a significant difference $(\mathrm{P}<0.0001$; Fig. 2D) existed among patients with edema of rounded and irregular shapes, with median OS times of 28.0 and 14.0 months, respectively However, multivariate analysis failed to confirm this significance. In addition, univariate analysis showed that 
Table II. Variables associated with OS in the entire cohort: Univariate analysis.

\begin{tabular}{|c|c|c|c|c|}
\hline \multirow[b]{2}{*}{ Variable } & \multirow[b]{2}{*}{ Number of cases } & \multicolumn{2}{|c|}{ OS, months } & \multirow[b]{2}{*}{ P-value } \\
\hline & & Median & $95 \% \mathrm{CI}$ & \\
\hline Total & 109 & 15.0 & $11.2-18.8$ & \\
\hline Gender & & & & 0.357 \\
\hline Male & 65 & 15.0 & $10.2-19.8$ & \\
\hline Female & 44 & 16.0 & $9.8-22.2$ & \\
\hline Age, years & & & & $<0.0001$ \\
\hline$\geq 60$ & 29 & 11.0 & 8.6-13.4 & \\
\hline$<60$ & 80 & 21.0 & $16.2-25.8$ & \\
\hline KPS & & & & $<0.0001$ \\
\hline$\leq 80$ & 42 & 13.0 & $10.6-16.8$ & \\
\hline$>80$ & 67 & 26.0 & $16.2-30.8$ & \\
\hline Chemoradiotherapy & & & & 0.004 \\
\hline Standard & 53 & 21.0 & $16.1-26.0$ & \\
\hline Non-standard & 56 & 12.0 & $8.1-15.9$ & \\
\hline Pathological grade & & & & $<0.0001$ \\
\hline Grade 3 & 44 & 28.0 & $8.0-54.0$ & \\
\hline Grade 4 & 65 & 12.0 & $9.5-14.5$ & \\
\hline PTE & & & & $<0.0001$ \\
\hline Minor & 34 & 44.0 & $20.4-67.6$ & \\
\hline Major & 75 & 14.0 & $12.3-15.7$ & \\
\hline Edema shape & & & & $<0.0001$ \\
\hline Rounded & 43 & 28.0 & $7.0-49.0$ & \\
\hline Irregular & 66 & 14.0 & $10.7-17.3$ & \\
\hline Necrosis & & & & $<0.0001$ \\
\hline None & 33 & 42.0 & $25.3-58.7$ & \\
\hline Mild & 43 & 14.0 & $8.2-19.8$ & \\
\hline Severe & 33 & 10.0 & $6.1-14.0$ & \\
\hline Cyst & & & & 0.593 \\
\hline None & 71 & 16.0 & $10.1-21.9$ & \\
\hline Small & 22 & 15.0 & $11.6-18.4$ & \\
\hline Large & 16 & 15.0 & $7.3-22.7$ & \\
\hline Enhancement & & & & $<0.0001$ \\
\hline No marked & 50 & 29.0 & $12.1-45.9$ & \\
\hline Marked & 59 & 13.0 & $9.4-16.6$ & \\
\hline Tumor crosses midline & & & & 0.762 \\
\hline No & 89 & 15.0 & $11.6-18.4$ & \\
\hline Yes & 20 & 21.0 & $9.0-33.0$ & \\
\hline Edema crosses midline & & & & 0.220 \\
\hline No & 77 & 17.0 & $12.7-21.3$ & \\
\hline Yes & 32 & 13.0 & $9.2-16.8$ & \\
\hline Size, cm & & & & 0.467 \\
\hline$<5$ & 65 & 15.0 & $12.4-17.6$ & \\
\hline$\geq 5$ & 44 & 19.0 & $10.7-27.3$ & \\
\hline
\end{tabular}

OS, overall survival; CI, confidence interval.

cysts $(\mathrm{P}=0.593)$, tumor crossing the midline $(\mathrm{P}=0.762)$, edema crossing the midline $(\mathrm{P}=0.220)$ and tumor size $(\mathrm{P}=0.467)$ were not significantly associated with the prognosis in malignant glioma. 
Table III. Statistically significant prognosis indicators evaluated by multivariate analysis in the entire cohort.

\begin{tabular}{lcrr}
\hline Variables & Hazard ratio & 95\% CI & P-value \\
\hline PTE & 2.337 & $1.089-5.015$ & 0.029 \\
Necrosis & 2.218 & $1.447-3.401$ & $<0.0001$ \\
Pathological grade & 2.066 & $1.150-3.713$ & 0.015 \\
Age, years & 1.954 & $1.137-3.358$ & 0.015 \\
KPS & 1.892 & $1.230-3.371$ & 0.023 \\
Chemoradiotherapy & 0.481 & $0.287-0.806$ & 0.005
\end{tabular}

PTE, peritumoral edema; KPS, Karnofsky performance status; CI, confidence interval.

Association between gender, age, KPS or pathological grade and patient prognosis. Gender did not affect the clinical outcome $(\mathrm{P}=0.357)$, but age $(\mathrm{P}<0.0001$; Fig. $2 \mathrm{~F})$, KPS $(\mathrm{P}<0.0001)$, pathological grade $(\mathrm{P}<0.0001$; Fig. $2 \mathrm{G})$ and post-operative chemoradiotherapy $(\mathrm{P}=0.004$; Fig. $2 \mathrm{H})$ were correlated with patient survival. Multivariate analysis indicated that age $(\mathrm{P}=0.015)$, KPS $(\mathrm{P}=0.023)$, pathological grade $(\mathrm{P}=0.015)$ and chemoradiotherapy $(\mathrm{P}=0.005)$ were all independent prognostic factors in the patients with malignant glioma.

\section{Discussion}

In the clinic, MRI is a routine examination for central nervous system diseases, and in particular, for the pre-operative diagnosis of glioma. Moreover, different signs shown by pre-operative MRI reflect the different biological behaviors of glioma. An improved understanding of the correlation between pre-operative MRI features and survival is therefore critical to clinical practice. The present study retrospectively analyzed several pre-operative MRI features, and found that PTE and necrosis were statistically significant unfavorable prognosis indicators affecting OS in patients with newly diagnosed supratentorial malignant glioma.

PTE, one of the main characteristics of malignant glioma, is a significant contributor to morbidity and mortality from glioma (15). The present study found that patients with major PTE exhibited a significantly worse OS time, and that PTE was an independent prognosis indicator. This may be associated with the fact that the glioma cells infiltrate the peritumoral area (20). Moreover, a recent study discovered a population of glioma stem cells infiltrating the area of PTE (21), and it has been found that these cells, which exhibit resistance to therapy, are the source of tumor recurrence (22-24). These data have provided convincing evidence that PTE is an indicator of an adverse prognosis in malignant glioma. However, a recent systematic review (14) suggested that the association between pre-operative PTE and survival in patients with glioma remains a controversial topic; one explanation may be that considerable heterogeneity exists in terms of patient clinical characteristics and the MRI technology used in these studies. Another factor that is inconsistent between the studies is the discrepancy in the measurement and classification of PTE. The present study also showed that patients with a rounded edema shape survived longer than those with an irregular edema shape, but multivariate analysis indicated that edema shape was not an independent predictor of prognosis. We speculate that edema extent may contribute to the result that edema shape affects patient survival, as this is consistent with the phenomenon observed in the clinic where PTE extent in patients with an irregular edema shape (such as a radial or finger-like shape) tends to be severe.

Necrosis is one of the pathological characteristics of malignant glioma. Previous studies have suggested that tumor necrosis extent is associated with a poor clinical outcome in malignant glioma $(8,25,26)$. The present study also found that tumor necrosis shown by pre-operative MRI was an independent unfavorable prognosis factor. This may be associated with the malignant biological behaviors of glioma cells in necrotic areas. The rapid cellular proliferation of malignant glioma causes nutrient imbalance, which leads to hypoxia and necrosis in tumor tissue. Necrotic areas are typically surrounded by pseudopalisading cells, which are relatively unique to malignant glioma and have long been considered as an unfavorable prognostic indicator (27). Moreover, the pseudopalisading cells show a more hypoxic nature (28), which not only is suspected to contribute to glioma development and progress (29), but also would promote tumor recurrence, and improve the invasion ability and resistance to radiochemotherapy (30).

It was previously believed that enhancement on pre-operative MRI was an independent predictor of survival in malignant glioma $(8,25)$. However, in the present study, the enhancement extent was associated with the OS of the patients with malignant glioma on univariate analysis, while it failed to retain its significance on multivariate analysis. One proposed explanation is that the enhancement of brain tumors mainly reflects the breakdown of the blood brain barrier and is affected by all processes that increase or decrease the abnormal permeability, regardless of the size and activity of tumor (31). Previous studies showed that cysts were associated with a better prognosis in low-grade glioma $(32,33)$. It is likely that this is since cyst formation represents more indolent neoplasm growth and therefore, improved survival (12). Nevertheless, the present study found that cysts did not affect the clinical outcome in patients with malignant glioma, which is in accordance with a previous large-scale study (12). The proposed hypothesis holds that cyst formation is associated with a disturbed blood brain barrier (34) or malignant transformation of a glioma (35). 
A

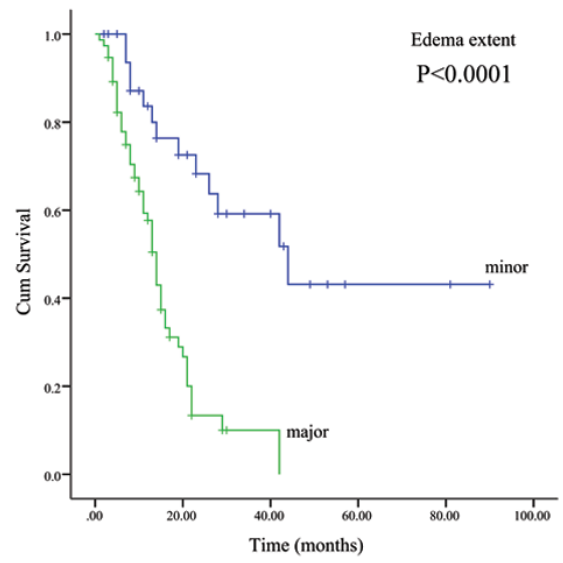

C

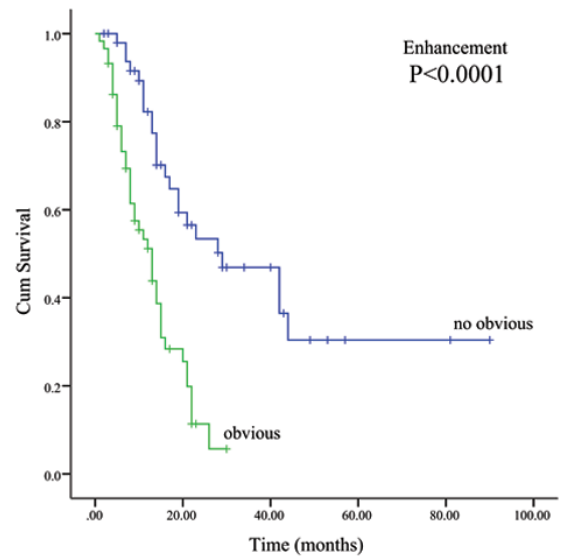

$\mathbf{E}$

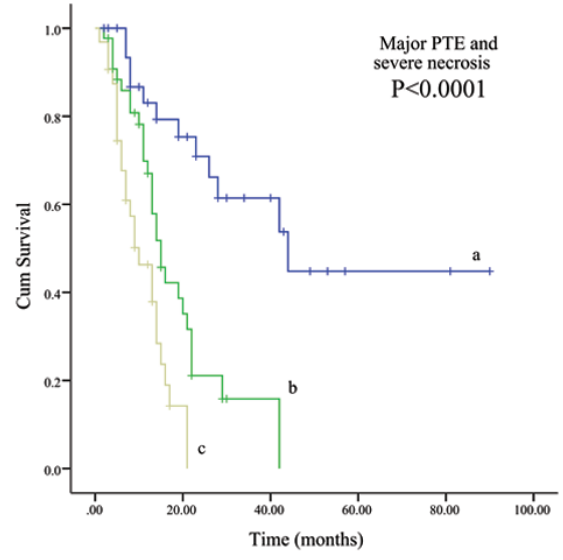

G

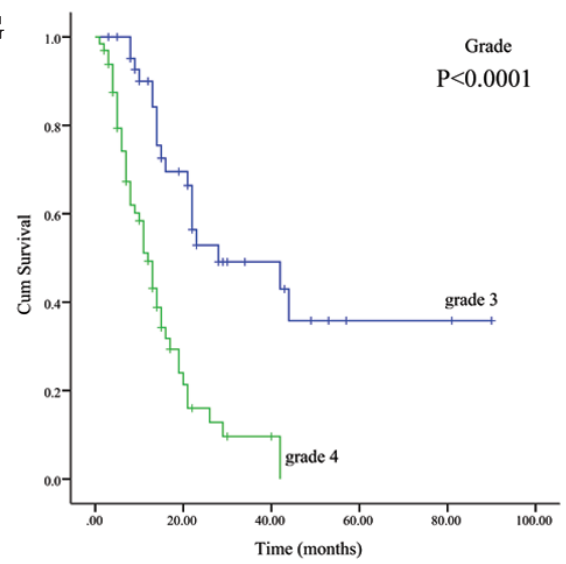

B

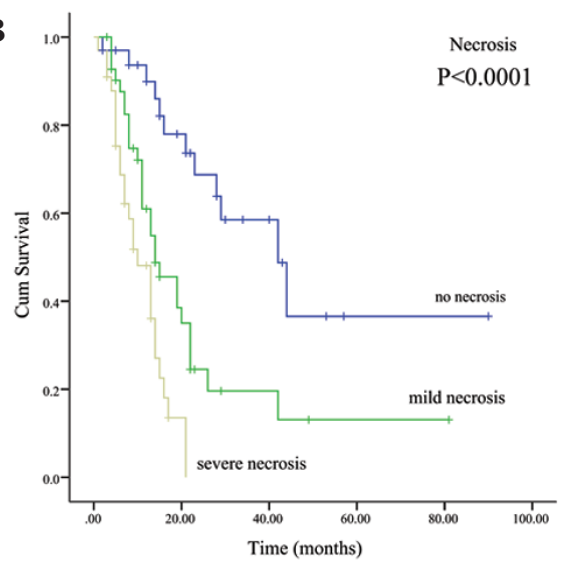

D
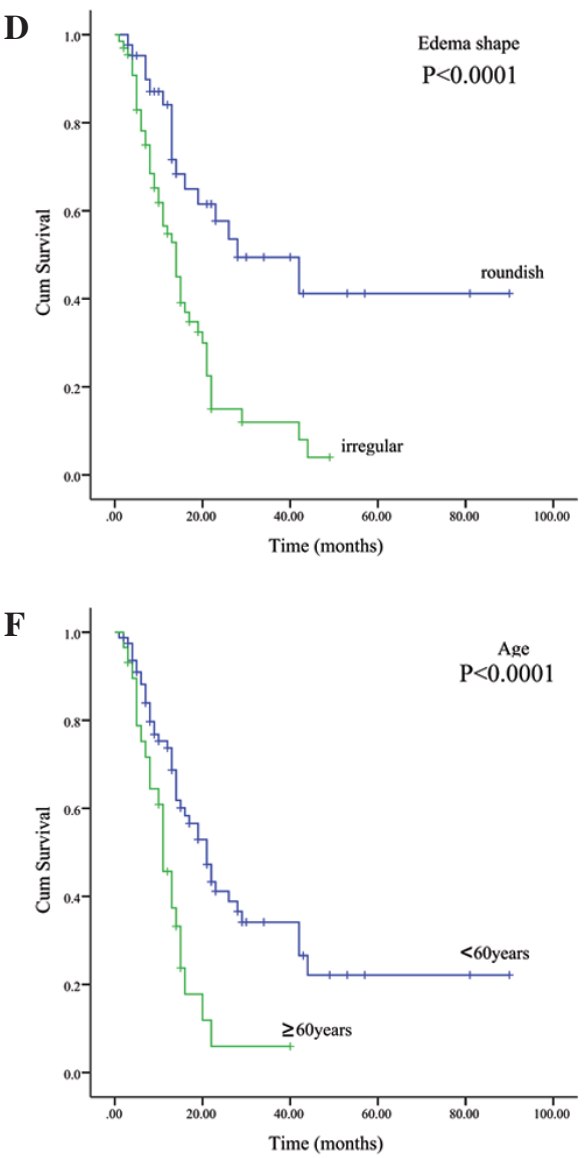

H

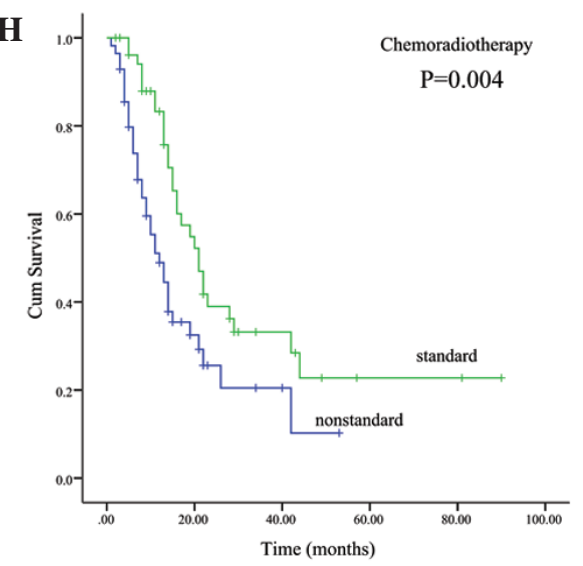

Figure 2. Correlations between (A) PTE, (B) necrosis, (C) enhancement, (D) edema shape, (E) major PTE and severe necrosis, (F) age, (G) grade and (H) chemoradiotherapy, and overall survival in the entire cohort (Kaplan-Meier curves). (E) a, the group with two unfavorable factors (major PTE and severe necrosis); b, the group with only one unfavorable factor (major PTE or severe necrosis); c, the group without major PTE and severe necrosis. PTE, peritumoral edema. 
However, the exact mechanism of cyst formation remains unknown and requires further research.

Additionally, it has been reported that age and pathological grade are independent predictors of prognosis in malignant glioma $(3,4)$, which is confirmed by the present results. We speculate that it may be due to the biological characteristics of patient at different age groups and the biological behaviors of glioma cells with different pathological grades. In the present study, post-operative standard chemotherapy plus radiotherapy was able to prolong the survival time of the malignant glioma patients, in accordance with the results of previous studies $(2,36)$. Therefore, we advocate that standard chemoradiotherapy for patients with malignant glioma should be actively pursued post-operatively.

However, a number of limitations that should be acknowledged exist in the present study. Firstly, this is a retrospective study, which may inevitably be subject to bias for information collection and patient selection. Moreover, the sample size is small in the study. Therefore, the association between pre-operative MRI features and prognosis in patients with supratentorial malignant glioma requires further analysis through large-scale and prospective studies, and particularly, the key molecular mechanisms of those independent predictors of survival require identification.

In conclusion, PTE and necrosis shown by MRI on pre-operative routine scans are independent indicators of an unfavorable prognosis, and patients with major edema plus severe necrosis exhibit a poorer prognosis, thereby indicating that PTE and extent of necrosis on routine MRI scans can be used to predict OS in patients with newly diagnosed supratentorial malignant glioma.

\section{Acknowledgements}

This study was supported by a grant from the National Natural Science Foundation of China (grant no. 30973083) and the Key Clinical Special Discipline Construction Program of Fujian (grant no. 2010-149).

\section{References}

1. Zhang X, Zhang W, Cao WD, Cheng G and Zhang YQ Glioblastoma multiforme: Molecular characterization and current treatment strategy (Review). Exp Ther Med 3: 9-14, 2012.

2. Yang LJ, Zhou CF and Lin ZX: Temozolomide and radiotherapy for newly diagnosed glioblastoma multiforme: A systematic review. Cancer Invest 32: 31-36, 2014.

3. Buckner JC: Factors influencing survival in high-grade gliomas. Semin Oncol 30: 10-14, 2003.

4. Yang LS, Huang FP, Zheng K, et al: Factors affecting prognosis of patients with intracranial anaplastic oligodendrogliomas: A single institutional review of 70 patients. J Neurooncol 100 113-120, 2010.

5. Schoenegger K, Oberndorfer S, Wuschitz B, et al: Peritumoral edema on MRI at initial diagnosis: An independent prognostic factor for glioblastoma? Eur J Neurol 16: 874-878, 2009.

6. Das P, Puri T, Jha P, Pathak P, Joshi N, Suri V, Sharma MC, Sharma BS, Mahapatra AK, Suri A and Sarkar C: A clinicopathological and molecular analysis of glioblastoma multiforme with long-term survival. J Clin Neurosci 18: 66-70, 2011.

7. Arshad H, Ahmad Z and Hasan SH: Gliomas: Correlation of histologic grade, Ki67 and p53 expression with patient survival Asian Pac J Cancer Prev 11: 1637-1640, 2010.

8. Hammoud MA, Sawaya R, Shi W, Thall PF and Leeds NE: Prognostic significance of preoperative MRI scans in glioblastoma multiforme. J Neurooncol 27: 65-73, 1996.
9. Pope WB, Sayre J, Perlina A, Villablanca JP, Mischel PS and Cloughesy TF: MR imaging correlates of survival in patients with high-grade gliomas. AJNR Am J Neuroradiol 26: 2466-2474, 2005.

10. Maldaun MV, Suki D, Lang FF, Prabhu S, Shi W, Fuller GN, Wildrick DM and Sawaya R: Cystic glioblastoma multiforme: Survival outcomes in 22 cases. J Neurosurg 100: 61-67, 2004.

11. Li WB, Tang K, Chen Q, Li S, Qiu G, Li SW and Jiang T: MRI manifestions correlate with survival of glioblastoma multiforme patients. Cancer Biol Med 9: 120-123, 2012.

12. Kaur G, Bloch O, Jian BJ, Kaur R, Sughrue ME, Aghi MK, McDermott MW, Berger MS, Chang SM and Parsa AT: A critical evaluation of cystic features in primary glioblastoma as a prognostic factor for survival. J Neurosurg 115: 754-759, 2011.

13. Pierallini A, Bonamini M, Osti MF, Pantano P, Palmeggiani F, Santoro A, Maurizi Enrici R and Bozzao L: Supratentorial glioblastoma: Neuroradiological findings and survival after surgery and radiotherapy. Neuroradiology 38 (Suppl 1): S26-S30, 1996.

14. Liu SY, Mei WZ and Lin ZX: Pre-operative peritumoral edema and survival rate in glioblastoma multiforme. Onkologie 36: 679-684, 2013.

15. Lin ZX: Glioma-related edema: New insight into molecular mechanisms and their clinical implications. Chin J Cancer 32: 49-52, 2013.

16. Louis DN, Ohgaki H, Wiestler OD, Cavenee WK, Burger PC, Jouvet A, Scheithauer BW and Kleihues P: The 2007 WHO classification of tumours of the central nervous system. Acta Neuropathol 114: 97-109, 2007.

17. Lin GS, Yang LJ, Wang XF, Chen YP, Tang WL, Chen L and Lin ZX: STAT3 Tyr705 phosphorylation affects clinical outcome in patients with newly diagnosed supratentorial glioblastoma. Med Oncol 31: 924, 2014.

18. Hartmann M, Jansen O, Egelhof T, Forsting M, Albert FK and Sartor K: Effect of brain edema on the recurrence pattern of malignant gliomas. Radiologe 38: 948-953, 1998 (In German).

19. Seidel C, Dörner N, Osswald M, Wick A, Platten M, Bendszus M and Wick W: Does age matter? A MRI study on peritumoral edema in newly diagnosed primary glioblastoma. BMC Cancer 11: 127, 2011.

20. Yamahara T, Numa Y, Oishi T, Kawaguchi T, Seno T, Asai A and Kawamoto K: Morphological and flow cytometric analysis of cell infiltration in glioblastoma: A comparison of autopsy brain and neuroimaging. Brain Tumor Pathol 27: 81-87, 2010.

21. Ruiz-Ontañon P, Orgaz JL, Aldaz B, Elosegui-Artola A, Martino J, Berciano MT, Montero JA, Grande L, Nogueira L, Diaz-Moralli S, et al: Cellular plasticity confers migratory and invasive advantages to a population of glioblastoma-initiating cells that infiltrate peritumoral tissue. Stem Cells 31: 1075-1085, 2013.

22. Zhang X, Zhang W, Mao XG, Zhen HN, Cao WD and Hu SJ: Targeting role of glioma stem cells for glioblastoma multiforme. Curr Med Chem 20: 1974-1984, 2013.

23. Chen J, Li Y, Yu TS, McKay RM, Burns DK, Kernie SG and Parada LF: A restricted cell population propagates glioblastoma growth after chemotherapy. Nature 488: 522-526, 2012.

24. Mangiola A, de Bonis P, Maira G, Balducci M, Sica G, Lama G, Lauriola L and Anile C: Invasive tumor cells and prognosis in a selected population of patients with glioblastoma multiforme. Cancer 113: 841-846, 2008.

25. Lacroix M, Abi-Said D, Fourney DR, Gokaslan ZL, Shi W, DeMonte F, Lang FF, McCutcheon IE, Hassenbusch SJ, Holland E, et al: A multivariate analysis of 416 patients with glioblastoma multiforme: Prognosis, extent of resection and survival. J Neurosurg 95: 190-198, 2001.

26. Pierallini A, Bonamini M, Pantano P, et al: Radiological assessment of necrosis in glioblastoma: Variability and prognostic value. Neuroradiology 40: 150-153, 1998.

27. Rong Y, Durden DL, Van Meir EG and Brat DJ: 'Pseudopalisading' necrosis in glioblastoma: A familiar morphologic feature that links vascular pathology, hypoxia and angiogenesis. J Neuropathol Exp Neurol 65: 529-539, 2006.

28. Brat DJ, Castellano-Sanchez A A, Hunter SB, Pecot M, Cohen C, Hammond EH, Devi SN, Kaur B and Van Meir EG: Pseudopalisades in glioblastoma are hypoxic, express extracellular matrix proteases and are formed by an actively migrating cell population. Cancer Res 64: 920-927, 2004.

29. Huang XD, Wang ZF, Dai LM and Li ZQ: Microarray analysis of the hypoxia-induced gene expression profile in malignant C6 glioma cells. Asian Pac J Cancer Prev 13: 4793-4799, 2012.

30. Oliver L, Olivier C, Marhuenda FB,et al: Hypoxia and the malignant glioma microenvironment: Regulation and implications for therapy. Curr Mol Pharmacol 2: 263-284, 2009. 
31. Brandsma D and van den Bent MJ: Pseudoprogression and pseudoresponse in the treatment of gliomas. Curr Opin Neurol 22: 633-638, 2009.

32. Jyothirmayi R, Madhavan J, Nair MK and Rajan B: Conservative surgery and radiotherapy in the treatment of spinal cord astrocytoma. J Neurooncol 33: 205-211, 1997.

33. Shibamoto Y, Kitakabu Y, Takahashi M, Yamashita J, Oda Y, Kikuchi $\mathrm{H}$ and Abe M: Supratentorial low-grade astrocytoma. Correlation of computed tomography findings with effect of radiation therapy and prognostic variables. Cancer 72 : 190-195, 1993
34. Adn M, Saikali S, Guegan Y and Hamlat A: Pathophysiology of glioma cyst formation. Med Hypotheses 66: 801-804, 2006.

35. Utsuki S, Oka H, Suzuki S, Shimizu S, Tanizaki Y, Kondo K, Tanaka S, Kawano N and Fujii K: Pathological and clinical features of cystic and noncystic glioblastomas. Brain Tumor Pathol 23: 29-34, 2006.

36. Stupp R, Mason WP, van den Bent MJ, Weller M, Fisher B, Taphoorn MJ, Belanger K, Brandes AA, Marosi C, Bogdahn U, et al: Radiotherapy plus concomitant and adjuvant temozolomide for glioblastoma. N Engl J Med 352: 987-996, 2005. 